\title{
Identificación de posibles prospectos geotérmicos utilizando álgebra de mapas en la porción central del Estado de Hidalgo, México
}

\author{
Identification of possible geothermal prospects using map algebra in the central part of Hidalgo \\ State, Mexico \\ Yerardi Méndez-Escamilla, Fred Y. Pérez-Corona, Ingrid Árcega-Santillán
}

Área Académica de Ciencias de la Tierra y Materiales, Universidad Autónoma del Estado de Hidalgo, Mineral de la Reforma, Hidalgo, 42184, México.

\begin{abstract}
:
Actually, in the Hidalgo State, geothermal energy is limited to use of balneology and there are few investigations directed to geothermal exploration, for this reason, a methodology is proposed that allows identifying possible geothermal prospects through a qualitative analysis with those variables That favor the accumulation of this type of energy. The study area is located in the central portion of the Hidalgo state, known as "geothermal play", it covers a surface of approximately 7,200 $\mathrm{km}^{2}$ and show a preferential northwest-southeast direction (NW-SE). The Weighted Sum method was used through map algebra to get an index of geothermal potential. In accord with results of this research, a map was designed that shows the possible areas with the lowest and highest geothermal potential, providing information to the geothermal industry that will be useful for future prospecting for this resource in the energy sector.
\end{abstract}

Keywords:

Geothermal energy, potential zones, geophysics, weighing, index.

\section{Resumen:}

Actualmente, en el estado de Hidalgo la geotermia está limitada al uso directo de la balneología y existen pocas investigaciones dirigidas a la exploración geotérmica, por esta razón, se propone una metodología que permite identificar posibles prospectos geotérmicos por medio de un análisis cualitativo con aquellas variables que favorecen la acumulación de este tipo de energía. El área de estudio se localiza en la porción central del estado Hidalgo, denominada como "play geotérmico", cubre una superficie de aproximadamente $7200 \mathrm{~km}^{2}$ y presenta una dirección preferencial noroeste-sureste (NW-SE). Se utilizó el método de Suma Ponderada por medio de álgebra de mapas para obtener un índice de potencial geotérmico. Con los resultados de esta investigación se diseñó un mapa que muestra las posibles zonas de menor y mayor potencial, aportando información a la industria geotérmica que será de utilidad para las futuras prospecciones de este recurso en el sector energético.

\section{Palabras Clave:}

Geotermia, zonas potenciales, álgebra de mapas, ponderaciones, índice.

\section{Introducción}

Actualmente, México se encuentra en la posición número seis de los países con campos geotérmicos de mayor capacidad en operación [12], contando con un total de 956 MW (megavatio) instalados en 5 plantas geotérmicas distribuidas en el país, esto según datos presentados en el Congreso Mundial de Geotermia llevado a cabo en Melbourne, Australia [4]. De estas plantas, cuatro se encuentran a servicio del gobierno, controladas por 
Comisión Federal de Electricidad (CFE) para satisfacer la demanda energética de las zonas aledañas a las plantas, y la planta restante se encuentra bajo orden privada para consumo de la empresa Grupo Dragón [5].

A pesar de estar en la lista de los 10 mejores países que aprovechan la geotermia para la generación de energía eléctrica, México aún es un país que invierte muy poco para el desarrollo de este tipo de energía, la cual, es renovable y sustentable. Es por eso que el Centro Mexicano de Innovación en Energía Geotérmica (CeMIEGeo) se ha encargado de generar información que facilite la búsqueda de recursos geotérmicos: mapas de flujo de calor. Uno de estos mapas muestra la categorización del gradiente geotérmico en provincias geotérmicas, basándose en el concepto de "play geotérmico" [18].

Un "play geotérmico" es un grupo de sistemas geotérmicos que comparten características geológicas similares, y que armonizan entre sí por que están controlados bajo la misma fuente de calor [16]. Con base en esta información se localizaron las zonas potencialmente geotérmicas en el estado de Hidalgo a partir de la delimitación de un "play geotérmico" de menor escala. Haciendo referencia a una menor escala con base en el catálogo de tipos de "play”, el cual utiliza áreas asociadas a fuentes anómalas de flujo de calor de dimensiones regionales como los grandes márgenes tectónicos activos, la dorsal meso atlántica es un ejemplo de ello [16].

Hasta el momento, la zona con la mayor cantidad de estudios de potencial geotérmico en el Estado de Hidalgo es el municipio de Tecozautla, zona donde estuvo instalada la primera planta geotérmica del país y de América en el año de 1959; nombrada Pathé [13]. Sin embargo, podría no ser el único lugar con este potencial. Existe la posibilidad de que esa zona corresponda únicamente a una pequeña parte de un gran yacimiento geotérmico aún sin estudiar. Por esta razón se realizó un análisis cualitativo que consistió en la identificación de variables favorecedoras para la configuración de sistemas geotérmicos, para este caso se utilizaron: la geomorfología, la geología, la crono-estratigrafía, el flujo de calor y la geofísica. Cabe mencionar que la metodología puede ocupar " $n$ " número de variables de acuerdo a la información a la que se tenga acceso, de tal manera que entre más detalle de variables se tengan mayor robustez y apego a la realidad tendrán los modelos que resulten del álgebra de mapas.

Dichas variables se conjuntaron por medio de Sistemas de Información Geográfica, utilizando el método de Suma Ponderada. Con este método se categorizaron las características de estas en clases, de modo que cada clase describiera la relevancia de cada una para la determinación de potencial geotérmico. Una vez re-clasificada cada variable, se asignaron los pesos o ponderaciones que definen el valor que tiene cada una sobre dicho potencial en la zona de estudio. Finalmente, con álgebra de mapas se adecuó la fórmula aritmética de la Media Ponderada con las variables reclasificadas y sus respectivas ponderaciones para obtener un índice de valores, que va de bajo a muy alto potencial geotérmico.

Este índice mostró las áreas de menor a mayor potencial, delimitando dos zonas potencialmente geotérmicas, una en el sector noroccidental y la otra en el sector suroriental del estado de Hidalgo. Estableciendo dichos sectores como los más prometedores para la exploración de recursos geotérmicos y permitiendo descartar la zona centro debido a su bajo potencial. Etimológicamente geotermia significa geo (Tierra) y thermos (calor) que en conjunto significan calor de la Tierra, esta energía se manifiesta en la superficie de distintas maneras; por ejemplo, en las aguas termales, géiseres, pozas de lodo, y fumarolas [6]. Esta energía térmica se origina a partir de dos fuentes, una es por la transferencia de calor desde el núcleo terrestre y la otra es por el calor generado durante el proceso de decaimiento radiactivo de algunos elementos [30]. La delimitación de la zona de estudio está basada en el concepto de "play geotérmico”, definido como condiciones geológicas que pueden soportar sistemas geotérmicos naturales o de ingeniería, donde la fuente de calor y los elementos geológicos que controlan la capacidad de transporte y almacenamiento del calor y los fluidos geotérmicos son los elementos clave [16]. Basado en el concepto anterior, se consideraron cinco variables elementales para delimitar las posibles zonas geotérmicas, estas son: la geomorfología, la geología, la crono-estratigrafía, el flujo de calor y la geofísica.

1. Marco geológico

El análisis geológico permite evaluar superficialmente sistemas geotérmicos, y al mismo tiempo inferir los medios de transporte de la energía calorífica por medio del reconocimiento estructural [7]. Por lo tanto, para comprender la relación y/o interdependencias entre los parámetros físicos y los operacionales en un yacimiento geotérmico, se debe comenzar con el estudio geológico.

\subsection{Geología Regional}

De manera general, el marco geológico de la zona de estudio se integra por una gran variedad de litologías y estructuras, dominando las de origen ígneo (figura 1) asociadas a la geodinámica de la Faja Volcánica Transversal Mexicana. Debido a la dimensión de la zona, esta se dividió en secciones para facilitar su descripción y análisis.

\subsubsection{Sección occidental}

Al norte, en esta sección afloran parcialmente calizas arrecifales de la Formación El Doctor, lutitas y calizas arcillosas pertenecientes a la Formación Soyatal, la cual se identifica en una pequeña porción de la zona cortada por la falla El Defay que mantiene una dirección NE-SW [24]. Cubriendo indistintamente a la unidad anterior se encuentra una secuencia volcánica de edad Oligoceno-Mioceno que varían de composición riolítica (definidas en el cerro Juárez) a basáltica (expuestas en el cerro Daxhie), conocidas como la Formación Las Espinas [24]. Esta sección está caracterizada por el vulcanismo de las calderas de Amealco y Huichapan. La litología está descrita en su origen como vulcanismo post- 
caldera, definidas por rocas de composición máfica e intermedia, siendo los aparatos volcánicos más sobresalientes, los cerros: La Cruz, El Cazadero, Colorado, Los caballos y Cerro Frío (ver figura 1); compuestos principalmente por secuencias andesíticas irregulares, emplazadas sobre derrames de basalto-andesita del plioceno tardío [28]. La estructura anular cercana a los aparatos volcánicos Peña Colorada, Siete Minas y Elefante (ver figura 1) se asocia a un lineamiento circular característico de las calderas o cuerpos intrusivos que tienen una geometría semi-circular. De igual modo, se identifican los domos resurgentes de la caldera de Huichapan (Hualtepec y Gexca) como productos de los últimos eventos explosivos de esta, los cuales extravasaron lavas de composición dacítica a riolítica de una edad aproximada de 4.2 ma [26] También se reconocen los cerros Xothe y Nopala como aparatos volcánicos de composición basáltica extravasada (ver figura 1).

\subsubsection{Sección central}

Las secuencias sedimentarias reconocidas en la poción occidental aún se presentan en la zona norte de esta sección, junto a algunos afloramientos del conglomerado polimíctico perteneciente a la Formación El Morro. Las secuencias ígneas están representadas por la extrusión de coladas de composición andesítica-basáltica del mioceno y plioceno, a la cual le sobre yace una sucesión vulcanoclástica de toba riolítica y dacítica conocida como Toba Donguinyó la cual, también es asociada a la actividad volcánica de la caldera de Huichapan [27]. Estas secuencias litológicas se reconocen en las elevaciones topográficas de mayor prominencia, como el cerro Xicuco reconocido como un antiguo aparato volcánico, el cerro El Picacho y el cerro Rincón de los Caballos (ver figura 1). En las cercanías de la capital del estado y del municipio de Actopan aflora una brecha volcánica dacítica del mioceno correspondiente a la Formación Zumate, la cual es reconocible en altos geomorfológicos como el cerro Las Monjas y cerro Plomosas. [23], reconociéndose al este de estos afloramientos vestigios de algunos aparatos volcánicos. El relieve volcánico, conserva rasgos característicos de su génesis geológica, (en los cerros: Las brujas, La Sotolera, El Picacho y El Chirerete) asociada al vulcanismo pleistocénico de la sierra de Las Navajas, la cual conserva un relicto de relieve semi-circular. La sierra de Las Navajas es un complejo volcánico principalmente riolítico que emplazo gran material vítreo y tobáceo en los alrededores [25].

Finalizando el registro litológico, se tienen depósitos piroclásticos dominados por derrames de lava y secuencias pumicíticas porosas depositadas durante el pleistoceno, las cuales cubren las edificaciones volcánicas conocidas como Cerro Redondo, Espíndola y Cubitos (ver figura 1), los cuales se conforman por andesitas y brechas extravasadas durante el oligoceno, mientras que otras se conforman en su totalidad de este material, por ejemplo, el cráter Tezontle y el cerro Los Montieles [25].

\subsubsection{Sección oriental}

La mayoría de los aparatos volcánicos se concentran en esta sección, ocurridos durante el plioceno y hasta el pleistoceno, los cuales caracterizan el vulcanismo del sector oriental de la Faja Volcánica Transmexicana [29]. Las rocas volcánicas más antiguas afloran en los cerros La Cantera y Ventoso, se trata de flujos de riolita, ignimbrita, secuencias de dacita, y brecha volcánica del mioceno. El vulcanismo del plioceno se reconoce en los vestigios volcánicos de los cerros: Verde, Napateco, Jagüey Chico, el Mirador, y La Campana; siendo este último el único de composición basáltica con intercalaciones de andesita, mientras que el resto están constituidos por derrames dacíticosriolíticos que evidencian el inicio de un episodio volcánico mucho más ácido y violento, ya que el alcance del material piroclástico cubre gran parte de esta sección [29]. Los cerros Molcajete, Paila (I y II), Tepetongo, Verde, Tepezala, Chato y Campanario son algunos de los domos monogenéticos formados por material piroclástico y lavas basálticas emplazadas en el mismo periodo que se originó el complejo volcánico Las Navajas, en el pleistoceno [29]. Los volcanes monogenéticos resultan ser de gran relevancia para comprender la relación entre la sismicidad, deformación y eventos de desgasificación en sistemas geotérmicos: eventos que favorecen vías térmicas pasivas [2]. Los conos cineríticos más destacados son los que comprende el campo volcánico de la caldera de Chilchicuautla, estos son Tecajete, Metepec, El Montito, Coatzetzengo, y las estructuras relictas de la misma caldera. Se caracterizan por secuencias piroclásticas depositadas durante el pleistoceno, siendo estas las evidencias de los últimos episodios volcánicos dentro de la zona [2019].

Cada uno de los edificios volcánicos descritos se muestran en el siguiente mapa (figura 1), el cual integra un esquema de menor escala al pie del grafico principal para localizar las secciones del play definidas anteriormente y las clases utilizadas en la metodología de álgebra de mapas.



Figura 1. Litología, geología estructural y principales edificios volcánicos de la zona denominada como "play geotérmico". 
Como se puede observar en la figura 1, la litología no es el único parámetro que compone a la geología; las fallas, alineaciones, entre otras componentes estructurales son importantes para comprender la estructura de un sistema geotérmico. De acuerdo con Jolie, Klinkmueller, Moeck, y Bruh [14], la geología estructural permite localizar áreas de manifestación térmica; sitios con mayor emanación y desgasificación de fluidos que ascienden a partir de diferentes fuentes de calor. Con base en esto, se puede observar que las fallas comprendidas en las áreas de influencia de los aparatos volcánicos permiten inferir que el sitio de estudio puede ser explorable, no sólo por su naturaleza geológica sino también por la dimensión de las componentes estructurales y su relevancia para la identificación de puntos de calor.

\section{Desarrollo experimental}

La zona de estudio se definió por medio de la localización de los principales sitios de interés térmico y las manifestaciones termales más conocidas del estado de Hidalgo, identificando así una tendencia lineal de la actividad geotérmica en dirección NW-SE (figura 2). Para establecer la amplitud de la zona de estudio, se digitalizaron los valores de flujo de calor obtenidos del mapa de provincias geotérmicas [17]. Con este mapa se definieron los valores de flujo de calor del Estado de Hidalgo y el alcance de las zonas con posible potencial geotérmico, la metodología propuesta tiene mayor robustez en sus resultados dependiendo del número de variables y detalle que estas tengan, por lo que en esta investigación se utilizaron variables de orden regional a las que se tuvo acceso, sin embargo, se tiene considerado integrar en una etapa posterior más variables con un nivel de detalle mayor.

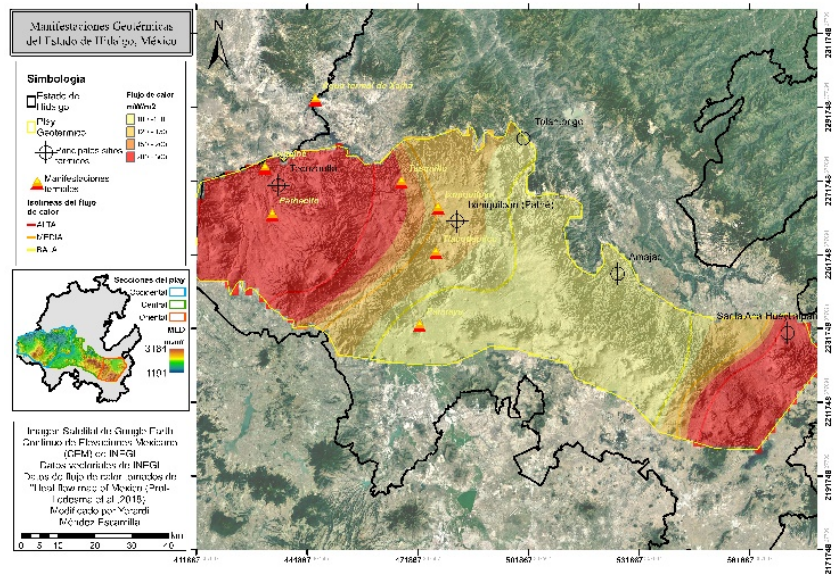

Figura 2. Mapa de las principales manifestaciones geotérmicas en el estado de Hidalgo.

Como se puede ver en la figura 2, la zona de estudio cubre un área extensa (cercana a los $7200 \mathrm{~km}^{2}$ ), presenta una geometría irregular con una longitud de $170 \mathrm{~km}$ aproximadamente y una amplitud entre los 30 y $70 \mathrm{~km}$, mostrando una dirección preferencial noroeste-sureste (NW-SE). Se localizan los sitios con manifestaciones térmicas superficiales en el estado de
Hidalgo, estos son: Tecozautla, Ixmiquilpan, Amajac y Santa Ana Hueytlalpan [11].

\section{Definición de variables \\ 1.1. Geomorfología}

La geomorfología de la zona de estudio está dominada por estructuras de origen ígneo, sierras volcánicas, domos, cráteres, volcanes extintos y calderas. Por medio de un análisis de estas geo-formas se infirieron los sitios más relevantes para la configuración de sistemas geotérmicos, ya que otorgan información sobre las fuentes de calor y su alcance. El análisis geomorfológico se basó en la identificación del tipo de relieve y su altura sujeta al nivel del mar [20].

\subsection{Geología}

El análisis del entorno geológico permite conocer la naturaleza de un sistema geotérmico [7]. Por lo tanto, para comprender la relación y/o las interdependencias entre los parámetros físicos y los operacionales en un yacimiento geotérmico, se debe comenzar con estudio geológico. El ambiente geológico de la zona de estudio está dominado por la geodinámica de la Faja Volcánica Transmexicana (FVT), estableciendo una variabilidad litológica a lo largo y ancho del "play geotérmico", en la porción occidental se reconocen afloramientos de edad OligocenoMioceno asociadas al vulcanismo post-caldera de composición máfica e intermedia. Mientras que en la zona centro comienza la transición de un vulcanismo intermedio a uno más acido, reconociendo secuencias piroclásticas de riolita-dacita y vidrio volcánico, Y, en la sección oriental se reconocen secuencias vulcanoclásticas mucho más acidas con intercalaciones de derrames máficos (basaltos), indicando la naturaleza bimodal de la FVT.

\subsection{Crono-estratigrafía}

Esta variable describe las épocas geológicas de mayor importancia para la acumulación de calor geotérmico con base en los eventos geológicos que las definen, principalmente los episodios volcánicos recientes, en otras palabras, con esta variable se identificó la edad de los afloramientos rocosos para establecer la temporalidad del vulcanismo, el cual indica la viabilidad térmica de los sistemas geotérmicos. Prol-Ledesma y Morán-Zenteno [18] denotan vulcanismo reciente aquel con edad $<=3$ ma (millones de años). Por lo tanto, se estableció al Holoceno, Pleistoceno y Plioceno como las 3 épocas de mayor relevancia para la ubicación de actividad volcánica reciente. Con este intervalo de tiempo se infirieron las áreas de acumulación y preservación de calor en la zona de estudio.

\subsection{Flujo de calor}

Con base en el mapa de flujo de calor y recursos geotérmicos de México [17], se digitalizaron las anomalías de calor de la zona de estudio, definiendo dos zonas altamente viables para la exploración de recursos geotérmicos, una en el sector noroeste y la otra al sureste. Cabe resaltar que esta información permitió conocer el calor disponible en la roca (flujo de calor), por medio de su densidad expresada en $\mathrm{mW} / \mathrm{m}^{2}$ [22]. 


\subsection{Geofísica}

Para el presente trabajo, la variable geofísica hace referencia a la magnetometría, método utilizado para la caracterización del “play geotérmico”. Los valores del campo magnético más importantes están asociados a las estructuras volcánicas de mayor dimensión, estas son las calderas de Huichapan y Chilchicuautla, mostrando anomalías de hasta $400 \mathrm{nT}$, definiéndolas como respuestas magnéticas intensas y por consiguiente favorables para la localización de rocas ígneas altamente magnéticas, las cuales son la fuente de calor más común en sistemas geotérmicos [26].

\section{Reclasificación}

El análisis cualitativo para la determinación de zonas potencialmente geotérmicas consistió en la selección de variables favorecedoras para la configuración de sistemas geotérmicos, estas variables son las descritas anteriormente: geología, geomorfología, crono-estratigrafía, flujo de calor y geofísica.
Las variables se reclasificaron con el fin de conjuntar en clases la relevancia para la determinación de potencial geotérmico de acuerdo a los elementos que las componen (Tabla 1), y así poder procesarlas por medio de álgebra de mapas a través de un algoritmo que emplea el fundamento teórico de las clases y ponderaciones con el objetivo de obtener un índice de valores. En la tabla 1 se muestra la información utilizada en la reclasificación de variables, la cual se obtuvo a partir de la clasificación de variables a partir del análisis de las propiedades que representan (elevación, litología, edad, energía térmica, y susceptibilidad magnética).

Tabla 1. Valores para la re-clasificación de las variables que conforman a un "play geotérmico" (Elaboración propia).

\begin{tabular}{|c|c|c|c|c|c|}
\hline Variable & Elementos & Propiedad & Valor & $\begin{array}{c}\text { Relevancia para la } \\
\text { determinación de posible } \\
\text { potencial geotérmico }\end{array}$ & Clase \\
\hline \multirow[t]{7}{*}{ Geomorfología } & Llanura & \multirow[t]{7}{*}{ Altura (msnm) } & $1191-1850$ & Medio & 2 \\
\hline & Pie de monte & & $1851-1899$ & Bajo & 1 \\
\hline & Ladera & & $1900-2020$ & Bajo & 1 \\
\hline & Montaña & & $2021-2650$ & Medio & 2 \\
\hline & Ruina Volcánica & & $2651-2800$ & Alto & 3 \\
\hline & Cráter & & 2801-2949 & Alto & 3 \\
\hline & Volcán & & $2950-3184$ & Alto & 3 \\
\hline \multirow[t]{8}{*}{ Geología } & Andesita-Basalto & \multirow{8}{*}{$\begin{array}{l}\text { Litología } \\
\text { relacionada con } \\
\text { vulcanismo } \\
\text { reciente }\end{array}$} & Muy Alto/Bajo & Alto & 3 \\
\hline & Basaltos & & Alto/Muy Alto & Alto & 3 \\
\hline & Andesita-Dacita & & Medio/Bajo & Medio & 2 \\
\hline & $\begin{array}{l}\text { Riolitas -Toba } \\
\text { riolítica }\end{array}$ & & Muy alto & Alto & 3 \\
\hline & $\begin{array}{c}\text { Toba riolítica -Toba } \\
\text { dacítica }\end{array}$ & & Muy Alto & Alto & 3 \\
\hline & Traquita & & Bajo & Bajo & 1 \\
\hline & Domos & & Medio & Medio & 2 \\
\hline & Calderas & & Alto & Alto & 3 \\
\hline \multirow[t]{3}{*}{$\begin{array}{c}\text { Crono- } \\
\text { estratigrafía }\end{array}$} & $\begin{array}{l}\text { Temprano } \\
\text { (Holoceno) }\end{array}$ & \multirow{3}{*}{$\begin{array}{l}\text { Vulcanismo } \\
\text { reciente } \\
<=3 \text { (millones } \\
\text { de años) }\end{array}$} & $<=0.0150 \mathrm{ma}$ & Bajo & 1 \\
\hline & Medio (Pleistoceno) & & $0.0150-3 \mathrm{ma}$ & Muy Alto & 4 \\
\hline & Tardío (Plioceno) & & $3-6 \mathrm{ma}$ & Alto & 3 \\
\hline \multirow[t]{4}{*}{ Flujo de calor } & $\begin{array}{c}\text { Anomalía de baja } \\
\text { amplitud }\end{array}$ & \multirow[t]{4}{*}{$\begin{array}{l}\text { Flujo de calor en } \\
\mathrm{mW} / \mathrm{m}^{2}\end{array}$} & $100-120$ & Bajo & 1 \\
\hline & $\begin{array}{l}\text { Anomalía de media } \\
\text { amplitud }\end{array}$ & & $120-150$ & Medio & 2 \\
\hline & $\begin{array}{c}\text { Anomalía de gran } \\
\text { amplitud }\end{array}$ & & $150-200$ & Alto & 3 \\
\hline & $\begin{array}{c}\text { Anomalía de enorme } \\
\text { amplitud }\end{array}$ & & $200-300$ & Muy alto & 4 \\
\hline \multirow{4}{*}{ Geofísica } & \multirow{4}{*}{$\begin{array}{l}\text { Campo magnético } \\
\text { anómalo reducido al } \\
\text { polo }\end{array}$} & \multirow{4}{*}{$\begin{array}{l}\text { Anomalía } \\
\text { reducida al polo } \\
\text { (nT) }\end{array}$} & $-744.6--125$ & Bajo & 1 \\
\hline & & & $-125.1--85$ & Medio & 2 \\
\hline & & & $-85.1-\quad-39$ & Alto & 3 \\
\hline & & & $-39.1-433$ & Muy alto & 4 \\
\hline
\end{tabular}


Es importante mencionar que la información obtenida de la variable geofísica requirió un proceso distinto al resto de las variables. Para el procesamiento de geofísica se utilizaron datos aero-magnéticos digitalizados de las cartas de magnetometría aérea del Servicio Geológico Mexicano (SGM).

En primer lugar, se delimitó la zona de estudio sobre esta información y se generó un archivo grid. El grid generado contiene el campo magnético anómalo residual de la zona de estudio. Con el fin de obtener una mejor definición de las anomalías magnéticas se aplicaron los filtros necesarios, dando como resultado una imagen con menor ruido y anomalías de mejor definición usando la Reducción al Polo (RP).

La RP simula llevar el área de estudio al polo magnético dónde la inclinación es igual a 90 grados y la declinación es de 0 grados, eliminando así la distorsión que existe del campo magnético en las diferentes latitudes de la Tierra y ubicando las anomalías magnéticas sobre las fuentes que las producen [9].

En la figura 3 se puede observar el campo anómalo magnético reducido al polo de la zona de estudio.

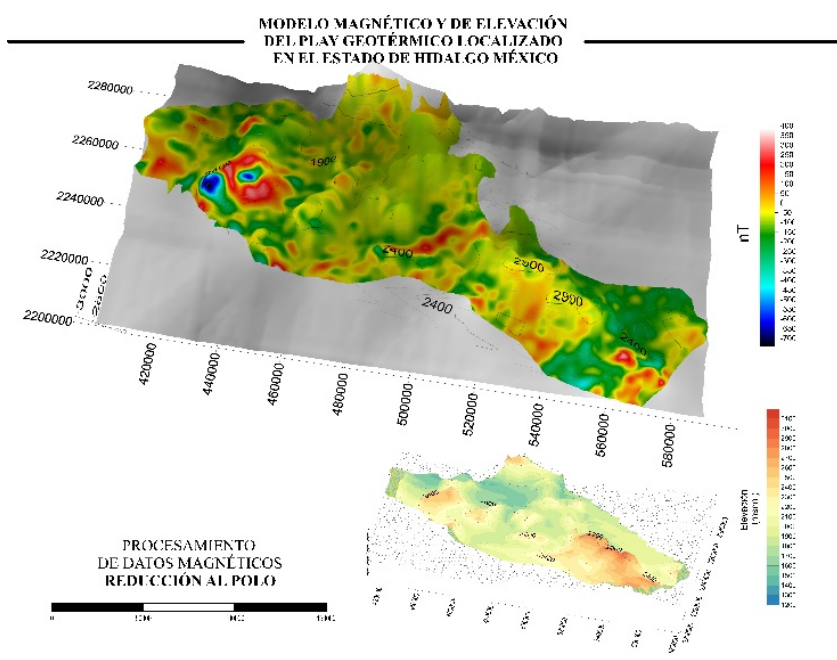

Figura 3. Modelo tridimensional del campo anómalo magnético reducido al polo de la zona de estudio.

En la figura 3 se representa un modelo tridimensional en donde se pueden identificar las anomalías de mayor susceptibilidad magnética, se reconocen en una amplitud de 50 a $400 \mathrm{nT}$ contenidas en el rango de colores naranjas a rojos. Estas definen bien los bordes de las estructuras que las originan permitiendo asociar las litologías y estructuras con los altos topográficos que muestra el modelo. Una vez reclasificados los datos magnéticos reducidos al polo y el resto de las variables, se convirtieron los archivos vectoriales a archivo ráster (rasterización) para posteriormente utilizarlos en la calculadora ráster, herramienta que permite concretar la técnica de álgebra de mapas.

\section{Método Suma Ponderada}

La técnica basada en algebra de mapas requiere la ejecución de un algoritmo matemático que permita conjuntar las variables a analizar, en este caso aquellas que conforman los recursos geotérmicos que definen a un "play geotérmico". Para esto, se definió la relevancia de cada variable por medio de una ponderación y una clasificación de valores basado en el método de Suma Ponderada. Este método es utilizado en análisis multicriterio o multivariable, en donde tiene la función de conjuntar distintas variables bajo la superposición de pesos ponderados o ponderaciones basados en la importancia relativa del tema analizado [15]. En su mayoría la selección de los pesos ponderados de las variables (cuales quieran los autores, porque también divergen) son por juicio del analista o por índices predeterminados por la naturaleza de la variable [8]; por lo que en la presente investigación se establecieron los pesos de las variables descritas anteriormente con base en la influencia de cada una de estas para la configuración de un sistema geotérmico. Al utilizar este método se tomó en cuenta que las variables requieren de una clasificación general (un valor que todas puedan representar) y una ponderación según su importancia para la determinación de un índice de potencial geotérmico [8].

La fórmula utilizada se describe a continuación:

$$
\mathrm{x}_{1} \mathrm{p}_{1}+\mathrm{x}_{2} \mathrm{p}_{2 \ldots \mathrm{n}} \mathrm{p}_{\mathrm{n}}=\sum_{\mathrm{k}=1}^{\mathrm{n}} \mathrm{x}_{k} \mathrm{P}_{k}
$$

Fórmula 1. Forma básica de la media aritmética ponderada

$$
\text { [21]. }
$$

x: variable

p: valor de la ponderación

n: número de variables

k: ubicación de los pesos o ponderaciones

De este modo, la suma de todas estas ponderaciones asociadas al total de variables analizadas es:

$$
N=\sum_{\mathrm{k}=1}^{\mathrm{n}} \mathrm{P}_{k}
$$

Fórmula 2. Suma de valores ponderados.

Para finalmente obtener la media ponderada en la siguiente fórmula:

$$
\bar{x}_{P}=\frac{\sum_{\mathrm{k}=1}^{\mathrm{n}} \mathrm{P}_{k}}{N}
$$

Fórmula 3. Media ponderada.

Por lo tanto, siguiendo el propio criterio y para aplicar la fórmula 3 , se asignaron los pesos o ponderaciones a las variables que conforman un "play geotérmico" (Tabla 2) tomando en cuenta que la suma de los pesos resulte en un total de 1 , factor numérico estándar que permite el rango de un índice de valores, o sea se normalizará el rango de análisis reclasificado [15]. 
En la tabla 2 se muestran las variables analizadas con sus respectivos pesos, con los cuales se completó la fórmula 4; donde $\mathrm{G}$ corresponde a geomorfología, $\mathrm{O}$ a geología, $\mathrm{E}$ a la crono-estratigrafía, $\mathrm{C}$ al flujo de calor y $\mathrm{F}$ a las anomalías aeromagnéticas derivadas de la variable geofísica.

Quedando la ecuación final de la siguiente manera:

$$
\bar{x}_{G O E C F}=(G * 0.1+O * 0.2+E * 0.2+C * 0.3+F * 0.2) / 1 .
$$

Fórmula 4. Ecuación para la determinación de un índice de potencial geotérmico basado en la fórmula de media ponderada.

Tabla 2. Ponderaciones de las variables.

\begin{tabular}{|ll|}
\hline Variable & Ponderación \\
\hline G & 0.1 \\
O & 0.2 \\
E & 0.2 \\
C & 0.3 \\
F & 0.2 \\
\hline
\end{tabular}

Este método permitió configurar las variables por medio de índices de potencial geotérmico; que van de 1 (menor potencial) a 4 (mayor potencial).

Una vez establecidos estos valores, se realizó el álgebra de mapas con el software QGIS (versión 3.8.1) siguiendo la estructura de la fórmula 4. Este proceso permitió la elaboración de un mapa geotérmico que delimita las zonas potenciales de dicha energía.

En la figura 4 se muestra este mapa, en él se pueden observar claramente los sitios que contienen este recurso y que antes se desconocía, por ejemplo, era más que conocida la existencia de manifestaciones geotérmicas en los municipios de Ixmiquilpan, Tecozautla y Huichapan, pero no se conocía el mismo valor geotérmico en los municipios de Zempoala, Singuilucan y Santiago Tulantepec, por mencionar algunos. Las zonas de menor potencial geotérmico cubren superficies relativamente pequeñas (zonas de color amarillo claro), por otro lado, las zonas de color naranja a color rojo que son las de mayor índice potencial cubren un área realmente grande.

En general se puede observar que los recursos geotérmicos son vastos, pero como se puede ver en el modelo tridimensional, a pesar de ser potenciales no se encuentran en sitios de fácil acceso, la topografía puede ser una desventaja para tener acceso a este recurso, o bien pueden estar involucrados otros factores que impidan el aprovechamiento de la geotermia en estos sitios, un ejemplo es la zona cercana al municipio de Acaxochitlán es un área de lomeríos y montañas medianamente elevadas, superficialmente parece ser un sitio de fácil acceso, pero no es posible asegurar esto sin una etapa de campo que lo compruebe. La verificación de vías de acceso para la comprobación de estos resultados es una etapa que no debe pasarse por alto.

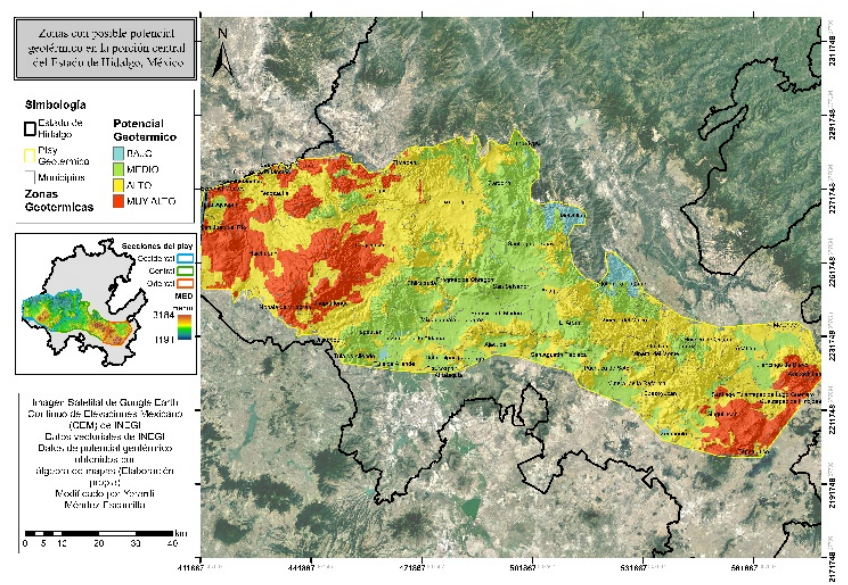

Figura 4. Mapa de potencial geotérmico en la porción central del Estado de Hidalgo, México

En la figura 4 se puede observar que el índice de potencial geotérmico obtenido muestra una mayor precisión en las zonas con altos valores de flujo de calor que el mapa mostrado en la figura 2.

\section{Conclusiones}

De acuerdo a los resultados obtenidos, el estado de Hidalgo preserva dos zonas altamente potenciales para el aprovechamiento de la geotermia, una al noroeste abarcando los municipios de Huichapan, Tecozautla, Tasquillo, Zimapán, Nopala, Chapantongo, Tula y Tepetitlán y la otra al sureste en Metepec, Tulancingo, Acaxochitlán, Santiago Tulantepec, Cuautepec de Hinojosa, Singuilucan, Tepeapulco, Zempoala y Epazoyucan. Ambas zonas están asociadas al vulcanismo del Plioceno, época donde los eventos volcánicos más significativos configuraron los campos geotérmicos más importantes de México (Humeros [10], Azufres [3] y Cerro Prieto [1]). La elaboración de un mapa de índices de potencial geotérmico ha permitido la discriminación de áreas poco relevantes para el desarrollo de la geotermia, facilitando la localización de zonas viables para la exploración y explotación de, conjuntando no solo características térmicas, si no también geológicas, geomorfológicas, crono-estratigráficas y geofísicas. Además, el modelo de alto relieve obtenido en la figura 3 permite definir los sitios con una topografía suave y menor accidentada, en la que sería más viable implementar una etapa de exploración directa para una futura explotación, por ejemplo, en la zona de valles de Santiago Tulantepec y Cuautepec de Hinojosa, o la zona de llanuras entre San Juan del Rio, Querétaro y Huichapan.

Los resultados obtenidos con la integración de las variables de geología, geomorfología, crono-estratigrafía, flujo de calor y geofísicas muestran una buena correlación con lo ya establecido en algunas zonas geotérmicas estudiadas, sin embargo, se tiene pensado en una etapa posterior integrar más variables en la metodología, para tener mayor robustez y detalle en la identificación de prospectos geotérmicos. 


\section{Conflicto de intereses}

Los autores declaran que no existen conflictos de intereses

\section{Referencias}

[1]Aguilar-Dumas, A. (2008). Análisis geológico-productivo enfocado a la exploración de la parte oriental del campo geotérmico de Cerro Prieto, BC. Geotermia,21(1),2-25.

[2] Albert, H., Costa, F., \& Martí, J. (2016). Years to weeks of seismic unrest and magmatic intrusions precede monogenetic eruptions. Geology. 44 (3): 211-214 pp. doi: 10.1130/G37239.1

[3] Arellano, V.M.,Torres, M.A.,Barragán, R.M.\& López, F.N.(2005). Evolución termodinámica (1982-2002) de los fluidos del campo geotérmico de Los Azufres, Mich. (México). Ingeniería Hidráulica en México, 2(1),7787.

[4] Bertani, R. (2015). Geothermal Power Generation in the World 20102014 Update Report, En Proceedings World Geothermal Congress, The Melbourne Declaration: Australia.GRC Bulletin. 14-15. Recuperado de: https://geothermal.org/PDFs/Articles/15MayJune.pdf

[5] Centro Mexicano de Innovación en Energía Geotérmica, (CeMIEGeo), (2014-2019). Geotermia en México. Recuperado de http://www.cemiegeo.org/index.php/geotermia-en-mexico

[6]Centro Mexicano de Innovación en Energía Térmica [CeMIEGeo]. (2014-2019). ¿Qué es la Geotermia? Recuperado de: http://www.cemiegeo.org/index.php/que-es-la-geotermia

[7] Daniilidis, A., M. Nick, H. \& Bruhn, D.F. (2020). Interdependencies between physical, design and operational parameters for direct use geothermal heat in faulted hydrothermal reservoirs. Geotermics. 86(20). doi: 10.1016/j.geothermics.2020.101806.

[8] Environmental Systems Research Institute. (2016). Conceptos del conjunto de herramientas de Superposición. Recuperado de https://desktop.arcgis.com/es/arcmap/10.3/tools/spatial-analysttoolbox/how-weighted-sum-works.htm

[9] Fanton, G., Martinez, M., Gimenez, M. (2015). Processing And Qualitative Data Analysis With Views Aeromagnetic Exploration Type Hydrothermal Deposits Lode Gold - La Rioja Province, Argentina. Geoacta. 39 (1). 30-50.

[10] González-Partida, E., Tello-Hinojosa, E.\& Pal-Verma, M. (2001). Interacción agua geotérmica-manantiales en el campo geotérmico de Los Humeros, Puebla, México. Ingeniería Hidráulica en México, 7(2),185-194.

[11] Gracia-Hernández, M. (2016). El turismo de salud en el estado de Hidalgo, México. Propuestas para consolidarlo. El periplo sustentable, (30), 157-179

[12] Gutiérrez-Negrín, L.C.A. (2019). Current status of geothermal-electric production in Mexico: IOP Conference Series. Earth and Environmental Science, 249, 1-11.

[13] Hiriart, G. (2017). Private Geothermal Opportunities in Mexico.GRC Transactions, 41(1), 1-8.

[14] Jolie, E., Klinkmueller, M., Moeck, I., Bruh, D. (2016). Linking gas fluxes at Earth's surface with fracture zones in an active geothermal field. Geology, 44 (3), 187-190 pp. doi: https: 10.1130/G37412.

[15] Marcano, A., Cartaya, S., Pacheco, H.\& Méndez, W. (2012). Estimación de pesos ponderados de variables para la generación de mapas de susceptibilidad a través de la Evaluación Espacial Multicriterio (EEM). I Congreso Venezolano de Ciencia, Tecnología e Innovación en el Marco de la LOCTI y del PEII. Ministerio del Poder Popular para Ciencia, Tecnología e Industrias Intermedias, Caracas, Venezuela.

[16] Moeck, I. S. (2014). Catalog of geothermal play types based on geologic controls. Renewable and Sustainable Energy Reviews, 37, 867882. doi: 10.1016/j.rser.2014.05.032.

[17] Prol Ledesma, R.M., Cruz, J.L., Torres-Vera, M.A., Espinoza-Ojeda, O., Carrillo, Juan., \& Membrillo-Abad, A. (2018). Heat flow map and geothermal resources in Mexico. Internagional e-jourrnals of maps, 2(2), 115, doi:10.22201/igg.25940694.2018.2.51.105.

[18] Prol-Ledesma, R.M., Morán-Zenteno, J. D. (2019). Heat flow and geothermal provinces in México. Geothermics, 78(1), 183-200.
[19] QGIS Development Team. (2019). QGIS Zanzibar (versión 3.8.1) [Software libre]. $\quad$ Descargado https://qgis.org/es/site/forusers/download.html

[20] Ramón-Melo, ML. (2017). Análisis Geomorfológico del Cerro San Cristóbal y Cerro Cumbre Las Tres Marías: Pachuca de Soto, Hidalgo, México. (Tesis de Licenciatura, publicada). Universidad Autónoma del Estado de Hidalgo. México.

[21] Rondero-Guerrero, C. (2010). Cálculo promedial. El caso de la media aritmética. Relime. 13 (4-2): 387-408.

[22] Sas, J., Osvald, M., Ramalho, E., \& Matos, J. (2018). Combined study of mineral deposits and deep geothermal for energy production or urban heating - Comparison between the Portuguese (Neves-Corvo) and the Hungarian (Recsk) case studies. Central European Geology. 61(2). 118135.

[23] Servicio Geológico Mexicano, SGM, 1995, Geológico-Minera, Carta Geológico-Minera Actopan, F14-D71, escala (1: 50 000): Pachuca, Hidalgo, SGM, 1da ed.

[24] Servicio Geológico Mexicano, SGM, 2001, Geológico-Minera, Carta Geológico-Minera Tasquillo, F14-C69, escala (1: 50 000): Pachuca, Hidalgo, SGM, 1da ed.

[25] Servicio Geológico Mexicano, SGM, 2007, Geológico-Minera, Carta Geológico-Minera Pachuca, F14-D81, escala (1: 50 000): Pachuca, Hidalgo, SGM, 1da ed.

[26] Servicio Geológico Mexicano, SGM, 2009, Geológico-Minera, Carta Geológico-Minera Huichapan, F14-C78, escala (1: 50 000): Pachuca, Hidalgo, SGM, 1 da ed.

[27] Servicio Geológico Mexicano, SGM, 2009, Geológico-Minera, Carta Geológico-Minera Mixquiahuala, F14-C89, escala (1: 50 000): Pachuca, Hidalgo, SGM, 2da ed.

[28] Servicio Geológico Mexicano, SGM, 2012, Geológico-Minera, Carta Geológico-Minera San Juan del Río, Querétaro, Hidalgo y Estado de México, F14-C77, escala (1: 50 000): Pachuca, Hidalgo, SGM, 2da ed.

[29] Servicio Geológico Mexicano, SGM, 2016, Geológico-Minera, Carta Geológico-Minera Tulancingo, F14-D82, escala (1: 50 000): Pachuca, Hidalgo, SGM, 1da ed.

[30] Zobin, V. M. (2017). The Seismic Signals Associated With the Natural Seismicity of Geothermal Structures Within Volcanic Environment. Introduction to Volcanic Seismology. (3 Ed), 497-517. 